\title{
Research on Sound Field Simulation Based Annoyance Evaluation Methodology Considering Mixed Noise Sources in Workshop
}

\author{
Yonghui Zhang ${ }^{1}$, Jinming Chen ${ }^{2}$, Bohan $\mathrm{Lv}^{1}$, Jihong Yan ${ }^{1}$ \\ ${ }^{1}$ Department of Industrial Engineering, Harbin Institute of Technology, Harbin, China \\ ${ }^{2}$ Beijing Satellite Environment Engineering Research Institute, Beijing, China
}

ABSTRACT: A quantitative mathematical model of mixed noise sources is proposed, which based on equivalent consecutive sound level $L_{A e q}$, aiming at overcoming the defect of A-weighted sound level, by workers' subjective response to noises cannot be represented. Multiple acoustic field Contour map is used to visualize the distribution of noise level and the noise level of a workshop can be calculated. Then the annoyance model combined with the predictive noise level is used to calculate the people's "subjective response" to noise. Finally, we take a FMS workshop as an example in order to illustrate and verify these proposed models, and it shows that the model can predict total annoyance well. This proposed method provides not only a new way to evaluate the noise influence of mechanical processing environment, but also a quatitative tool for assessing the compatibility of equipment and environment.

KEYWORD: Workshop; $L_{\text {Aeq }}$; Acoustic Field; Noise Annoyance; Simulation; Quantitative Analysis

\section{INSTRUCTIONS}

Noise is one of the main occupational hazard of mechanical processing, it is easy to be ignored when noise pollution occurs in the workshop due to the fact that industrial noise pollution is less widespread than transportation noise and air pollution. Locally, industrial noise can cause great annoyance. Evaluating noise correctly helps to improve working conditions for workers. Research on quantitative evaluation of noise of workshop and analysis of the effects of noise exposure on people has been very little studied to date. Noise annoyance is generally used to describe the negative feelings associated with noise according to the European Commission (Zaheeruddin, Z., et al. 2006). The domestic study on noise annoyance effect is still at the initial stage. Research carried out on noise annoyance due to combined noises has mainly focused on transportation (road traffic, railways, aircraft and tramways) noises (Liang, Y., et al. 2012).Thus, both laboratory and field studies are needed to better assess noise annoyance due to industrial noise. Research on the methodologies for simulation of sound field and evaluation of annoyance from mixed noise sources for workshop are of significance for achieving better work environment and production efficiency.
Aiming at conducting acoustic field's quantitative analysis and visualizing the negative feelings annoyance associated with combined noise souces, this research involved an effective approach to analyze environmental noise quantitatively combined with common acoustic index $L_{\text {Aeq }}$ and an evaluation method of noise annoyance from combined noise sources. Firstly, a mathematical model from combined noise sources is established. Then the procedure of numerical simulation and quantitative analysis on environmental acoustic field of workshop is presented. Secondly, several total annoyance models are reviewed, the energy summation is found to be the most practically appropriate model in this study. Finally, combined with Multiple acoustic field model and annoyance calculation formula, we transform the multiple acoustic field Contour Map into noise annoyance of mixed sources Contour Map, realized the visualization of workers' subjective response to noise in a machining workshop.

\section{SOUND FIELD MODELLING AND ANNOYANCE EVALUATION METHOD}

\subsection{Sound Field Modelling based on $L_{\text {Aeq }}$}

A physical model of workshop based on a Flexible Manufacture System (FMS) workshop is established 
with attenuation caused by walls and decreasing with propagation distance increased in air. $L_{A e q}$ is the index that indicates noise intensity via weighted calculation throughout the working day in each period of time, therefore the results of sound field simulation represent the true extend of workers exposed to noise in an entire working day. In this paper, the noise evaluation index is modified by the impact of continuous equivalent sound level $L_{\text {Aeq }}$ for shop workers, the establishment of mathematical model of multiple sound sources field is built to realize quantitative analysis of the annoyance caused by environmental noise.

Figure1 shows the layout of a Flexible Manufacture System (FMS), which is an assembling workshop at our lab. The workshop is with the size of $12 \mathrm{~m} \times 12 \mathrm{~m} \times 5 \mathrm{~m}$, and there are $n$ equipment Machines $\left(M_{i}, i=1, \ldots n\right)$ inside with the noise intensity $K_{1}, K_{2}, \cdots, K_{n}$ when the machine is running, and there are 8 locations $\left(A_{j}, j=1, \ldots, 8\right)$ where we collected noise data.

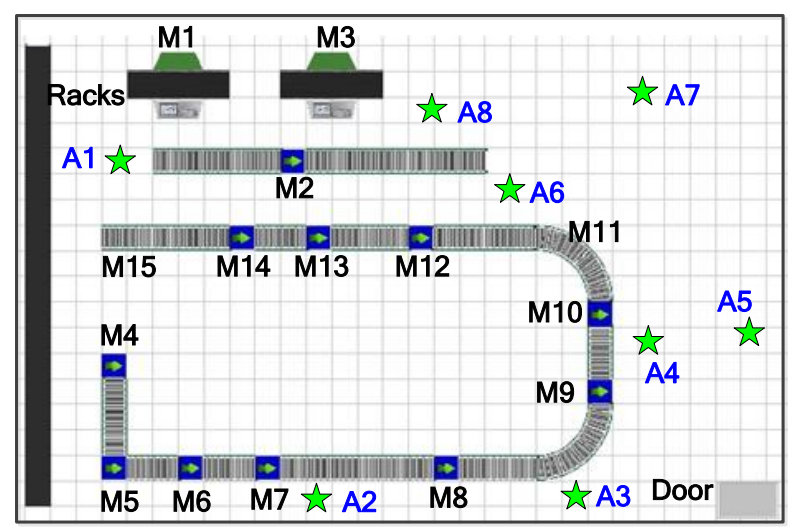

Figure 1. Layout of the FMS workshop

Boundary conditions:

(1) Each equipment is treated as a point sound source in a workshop;

(2) Ignoring the vibration of machine tools shell; the actual noise intensity will attenuate in the process of noise propagation in air.

The procedures for modeling noise sound field are as follows:

Assume there are $n$ noise sources with the three dimensions location information $(x, y, z)$.

$M_{i}=\left(x_{i}, y_{i}, z_{i}\right) \quad i=1,2, \cdots, n$

Where $i$ represents the serial number of $n$ machines, and $\left(x_{i}, y_{i}, z_{i}\right)$ indicates the location of each machine/sound source.

There is a test point $M_{0}(a 0, b 0, c 0)$, then the distance between $M_{0}$ and $n$ sound sources can be calculated by equation (2).
Distance $_{i}=\sqrt{\left(x_{i}-a_{0}\right)^{2}+\left(y_{i}-b_{0}\right)^{2}+\left(z_{i}-c_{0}\right)^{2}}$

The mixed noise of $n$ noise sources at point $M_{0}$ is described by equation (3).

$$
L E S_{i}=K_{i}-\Delta+10 \times \lg S-20 \times \lg \left(\text { Distance }_{i}\right)-14
$$

Where $K_{i}$ represents the actual noise intensity of the each machine. $S$ represents the surface area of building exterior wall and $\Delta$ represents octave band sound insulation quantity from internal system to the outside free field.

With noise attenuation considered, we assumed that the sound reduction index of the wall is $R_{1}$, the sound reduction index of the window is $R_{2}$ and the sound reduction index of the door is $R_{3}$. We can get the $R_{k}, k=1,2,3$ listed in table 3-2 according to (Chang Ruiqing, et al. 2001). Then, the actual noise intensity in the test point $M_{0}$ caused by $n$ sound source is expressed as follows:

$$
L E S D_{i}=L E S_{i}-\min \left\{R_{1}, R_{2}, R_{3}\right\}
$$

According to Technical Guidelines for Noise Impact Assessment (HJ 2.4-2009) and the theory of Sound Pressure Level calculation, the accumulative total noise intensity of $n$ sound source in the test point $M_{0}$ can be expressed as follows:

$$
L_{E Q}=10 \times \lg \left(\frac{1}{T} \sum_{i=1}^{n} T_{i} \times 10^{0.1 \times L E S D_{i}}\right)
$$

Where $L_{E Q}$ is the total $L E S D_{i}$ from the combined sources, $L E S D_{i}$ is the sound source $i$ in the equivalent sound level of contribution to the prediction of $M_{0}$ point.$T_{i}$ is the total running time of $i$ machine in the $T$ period, $T$ is the total prediction time(second).

$$
T=\sum_{i=1}^{n} T_{i}=\sum_{i=1}^{n} 252 \times t_{i}
$$

Where $t_{i}$ is the processing time of $i$ machine (see, Table 1), The daily production of this workshop is 252 parts.

\subsection{Evaluation Method of Annoyance}

Previous research on human health and environmental effects of industrial noise mainly based on noise monitoring of industrial equipment value or environment from acoustic point monitoring value as an evaluation criterion, this method has its advantages as on time, comparable to a certain extent, however there are obvious limitations for the fact that this method can not accurately predict 
subjective response to the noise level. In order to evaluate noise annoyance due to noise combination, researchers have attempted to model total annoyance. Seven classical models of total annoyance are reviewed (Pierrette, M. et al. 2012), the energy summation is found to be the most practically appropriate model since it is useful in the situation in which the multiple sources sound field model is built. In this study, energy summation model is considered. It is briefly described below considering mixed noise sources.

$\alpha_{A}=f\left(L_{E Q}\right)$

Where $\alpha_{A}$ is the annoyance response to the combined noise sources.

This model is based on the assumption that the annoyance caused by combined noise sources can be predicted by the total energy.

\section{VERIFICATION AND APPLICATION}

\subsection{FMS workshop and data collection}

According to the Measurement of Noise produced by Industrial Enterprises (GBJ122), we use the sound pressure meter to collect the noise data. Figure 3 shows the monitoring point $\left(A_{i}\right)$. Monitored noise data are listed in Table 1.

Table 1. Noise monitoring data and processing time .

\begin{tabular}{|l|l|l|l|}
\hline ID & Machine & Noise $(\mathrm{dB})$ & $t_{i}(\mathrm{~s})$ \\
\hline M1 & Lathe & 103.9 & 20 \\
\hline M2 & Milling machine & 86.9 & 10 \\
\hline M3 & Manipulator & 100.6 & 30 \\
\hline M4 & Manipulator & 96.2 & 10 \\
\hline M5 & CNC & 84.6 & 35 \\
\hline M6 & Feeding unit & 97.3 & 10 \\
\hline M7 & Blanking unit & 92.9 & 10 \\
\hline M8 & Affixing unit & 95.7 & 5 \\
\hline M9 & Pining unit & 97.8 & 5 \\
\hline M10 & Analog signal unit & 92.8 & 10 \\
\hline M11 & Reversing unit & 90.2 & 10 \\
\hline M12 & Image recognition & 91.1 & 15 \\
\hline M13 & Check unit & 90.4 & 10 \\
\hline M14 & Sorting unit & 93.3 & 15 \\
\hline M15 & Stacking machine & 92.0 & 20 \\
\hline
\end{tabular}

\subsection{Results and discussion}

Sound Field Simulation is implemented using Matlab, We use the data collected in the first step as the input, and visualize the output by a colored 3D picture (see, Figure2).

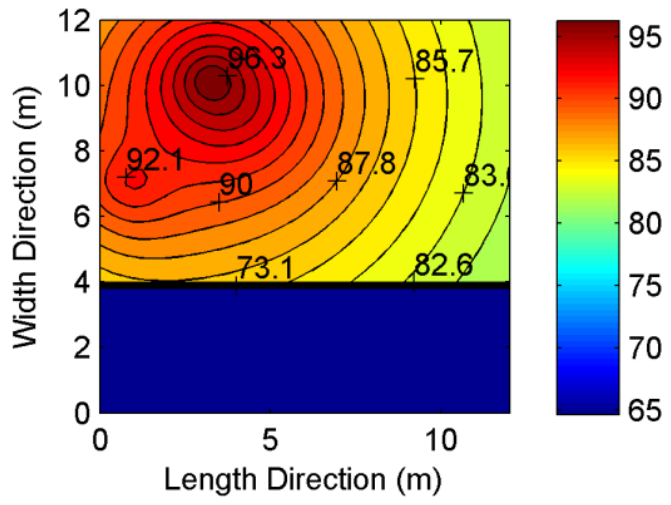

Figure 2. Multiple acoustic field Contour Map

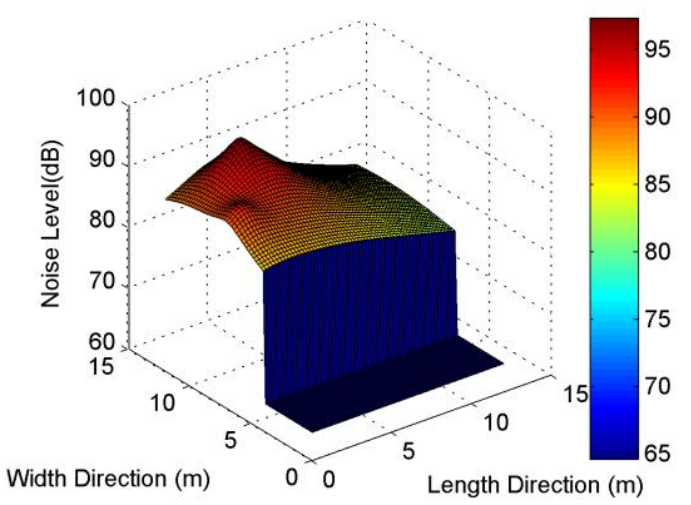

Figure 3. Multiple acoustic field 3D map

Figure 2 shows the acoustic pressure Contour map, where the range and degree of the noise level can be distinguished obviously. It helps people to figure out the safe operation range under different noise limit values and eventually protect the workers from the noise harm. It shows that sound pressure level in this workshop is $79.8 \sim 102 \mathrm{~dB}(\mathrm{~A})$, which means that the noise is harmful to workers, the reason why we build the noise annoyance is obvious here. We compare the measured value and calculated value on some typical positions (where workers do operation regularly) in Table 2.

Table 2 indicates the maximum relative error is $6.76 \%$, which illustrates the validity and accuracy of the proposed model, and it is also the base of the noise annoyance model. According to the Sanitary Standard for the Design of Industrial Enterprises (GBZI2002), the noise limit is listed in Table 3.

Table 2. Comparison of monitored and simulated noise

\begin{tabular}{|c|c|c|c|}
\hline Sites ID $(\mathrm{m})$ & Monitoring dB (A) & Simulation $\left(L_{E Q}\right)$ & $\mathrm{RE}$ \\
\hline $\mathrm{A} 1$ & 95.1 & 98.4 & $0.40 \%$ \\
\hline $\mathrm{A} 2$ & 97.1 & 95.4 & $2.52 \%$ \\
\hline $\mathrm{A} 3$ & 95.9 & 92.7 & $3.34 \%$ \\
\hline $\mathrm{A} 4$ & 92.8 & 93.4 & $0.65 \%$ \\
\hline $\mathrm{A} 5$ & 88.9 & 90.5 & $1.80 \%$ \\
\hline $\mathrm{A} 6$ & 91.2 & 95.5 & $4.71 \%$ \\
\hline $\mathrm{A} 7$ & 85.6 & 91.4 & $6.76 \%$ \\
\hline $\mathrm{A} 8$ & 92.1 & 96.3 & $4.56 \%$ \\
\hline
\end{tabular}


As the results listed in Table 2, Simulation results $L_{E Q}$ of typical work sites $A_{5}$ is $90.5 \mathrm{~dB}$, According to the Sanitary Standard for the Design of Industrial Enterprises (GBZI2002)-occupational Exposure Limits for noise, work time limit of work sites $A_{5}$ is 1.8 hour for a day. Noise level of work sites and work time limit can be obtained by the model. Results of noise analysis using this model can provide a reference for labor protection. Finally, we use the Simulation $L_{E Q}$ data in the second step as the input of the Noise annoyance model, and visualize the output by a colored 3D picture (see, Figure.4).

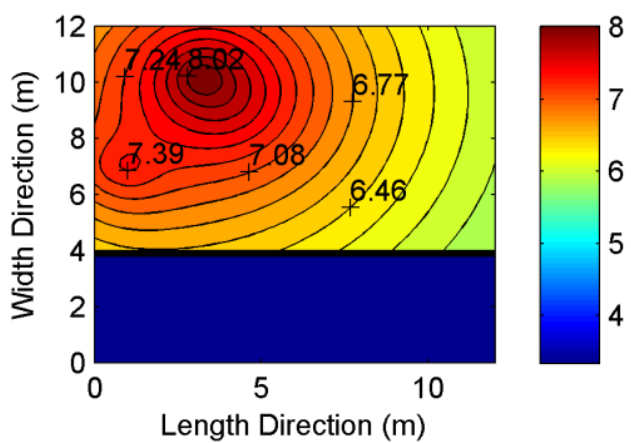

Figure 4. Noise annoyance of mixed noise sources Contour Map

From Figure 4, workers' response to noise can be seen intuitively. Noise annoyance value is between 3.49 8.02. Noise Annoyance quantitative analysis method will assist to the development of operational standards in the noisy environment. Based on the quantitative annoyance analysis, we can fully consider the influence of the equipment noise in planning the workshop standards, reasonably plan the work area and minimize noise, provide a relatively good working environment for workers in the workshop.

Table 3. Occupational Exposure Limits for noise

\begin{tabular}{|c|c|c|}
\hline Indicator & Time Limit & Noise level dB(A) \\
\hline & $24 \mathrm{~h}$ & 80 \\
exposure & $16 \mathrm{~h}$ & 82 \\
limit & $8 \mathrm{~h}$ & 85 \\
value & $4 \mathrm{~h}$ & 88 \\
& $2 \mathrm{~h}$ & 91 \\
& $1 \mathrm{~h}$ & 94 \\
\hline Maximum limit & \multicolumn{2}{|c|}{$115 \mathrm{~dB}(\mathrm{~A})$} \\
\hline \multirow{2}{*}{ crossoverate } & it allows noise limit increased 3dB \\
& \multicolumn{2}{|c|}{ when exposure time halve } \\
\hline
\end{tabular}

\section{CONCLUSIONS}

Based on the theory of the sound field attenuation, superposition and the property of sound, this paper proposes a mathematical model for annoyance quantitative analysis under mixed noise environment. The noise of different operation points can be calculated at the same time by using the proposed model and the visualization technique is applied to observe the distribution of noise in the workshop. The quantitative analysis of multiple acoustic sources provides an effective way to estimate the noise distribution constantly and scientifically. A noise annoyance model is proposed to reveal the human being's subjective response to noise. We combined the mathematical model and the noise annoyance model and delivered noise annoyance Contour map, which reflects the "subjective response" of the workers to noise in different location in a workshop intuitively.

\section{ACKNOWLEDGMENT}

This work is funded by National Science Foundation (\#71271068).

\section{REFERENCES}

[1] Zaheeruddin, Z., Jain, V. K., \& Singh, G. V. (2006). A fuzzy model for noise-induced annoyance. Systems, Man and Cybernetics, Part A: Systems and Humans, IEEE Transactions on, 36(4), 697-705.

[2] Liang, Y., Ke-An, C., \& Stoop, R. (2012). Noise annoyance from a mixture of multiple single sources: rating and prediction.

[3] Chang Ruiqing, Han Yu. (2001). Study on the effect of wall on noise abatement. Environment Protection Department of Baotou Steel (Group) Corporation,Baotou, 27(1), 72-75.

[4] Pierrette, M., Marquis-Favre, C., Morel, J., Rioux, L., Vallet, M., Viollon, S., \& Moch, A. (2012). Noise annoyance from industrial and road traffic combined noises: A survey and a total annoyance model comparison. Journal of environmental psychology, 32(2), 178-186.

[5] Taylor, S. M. (1982). A comparison of models to predict annoyance reactions to noise from mixed sources. Journal of Sound and Vibration, 81(1), 123-138. 